\title{
PSICOLOGIAEDUCACIONALNOSCURSOSDELICENCIATURA: AMOTIVAÇÃODOSESTUDANTES
}

\author{
Sueli Édi Rufini Guimarães ${ }^{1}$ \\ José Aloyseo Bzuneck ${ }^{2}$ \\ Samuel Fabre Sanches ${ }^{2}$
}

\section{Resumo}

Estudo de natureza exploratória, investigou as orientações motivacionais e o envolvimento na disciplina Psicologia Educacional de 246 alunos de cursos de licenciatura da Universidade Estadual de Londrina. Foram administradas a todos os participantes escalas de avaliação da motivação intrínseca e extrínseca (Work Preference Inventory) e do esforço alegado na disciplina. Os participantes também responderam a um questionário referente aos dados demográficos e à intenção de atuar no ensino. Análises de variância e estudos correlacionais revelaram diferenças significativas entre as medidas empregadas e as variáveis demográficas investigadas. Os resultados são discutidos em termos de sua contribuição para compreensão aprofundada dos padrões motivacionais dos estudantes e para a melhoria do ensino da disciplina Psicologia educacional em cursos de formação de professores.

Palavras-chave: motivação e aprendizagem; motivação intrínseca

\section{Educational Psychology inTeaching Students: Their Motiuation}

\begin{abstract}
This study investigated the motivational orientations and involvement in Educational Psychology courses of 246 undergraduate majoring in teaching students at Londrina State University (Brazil). Measures of intrinsic and extrinsic motivation (WPI) and scales for assessment of reported effort, as well as a questionaire regarding demographic and self-perception of achievement data were administred to all the participants. Analyses of Variance and correlational studies revealed significant differences between the measures employed and the demographic variables invetigated. Findings are discussed in terms of their contribuition not only for a deeper understanding of the students motivational patterns, but also for the improvement of Educational Psycology courses for undergraduates teaching students.
\end{abstract}

Key-words: motivation and learning; intrinsic motivation

\section{INTRODUÇÃo}

Os cursos de formação de professores para o ensino fundamental e médio, existentes nas diversas instituições de ensino superior do país são, de modo geral, organizados em disciplinas específicas da área de origem e em disciplinas denominadas pedagógicas. Tradicionalmente, a Psicologia Educacional tem composto o quadro por ser uma disciplina acadêmica, cujo campo de pesquisas mantém relações estreitas com a prática educacional. A natureza e a especificidade dos conteúdos, assim como os problemas nela abordados, limitam sua interação com as demais disciplinas pedagógicas (Didática, Estrutura e Funcionamento do Ensino, Metodologia e Prática de Ensino). Este distanciamento, repetidamente apontado e criticado, não impede que os alunos percebam a Psicologia Educacional como integrada às demais disciplinas de formação, ou seja, compartilhando os mesmos vínculos com o ensino (Parra, 1984; Bzuneck, 1999).

Doutoranda da Universidade Estadual de Campinas

${ }_{2}$ Doutor e docente da Universidade Estadual de Londrina 
Observa-se entre os estudantes dos cursos de licenciatura uma motivação bem diversificada para aprender os conteúdos das disciplinas pedagógicas. Ao lado de alguns, preocupados em aprofundar o nível de conhecimentos ou em atingir uma fundamentação adequada para o desempenho de sua futura função, estão presentes alunos apáticos, pouco dispostos a participar das atividades propostas, outros preocupados apenas com a obtenção de notas ou com a conclusão do curso e, outros, ainda, com uma evidente intenção de trabalhar ou comparecer às aulas o mínimo solicitado para garantir sua aprovação.

Compreender os fatores relacionados ao envolvimento dos estudantes com a aprendizagem escolar é uma tarefa considerada complexa, tendo em vista a pluralidade dos elementos presentes na situação. Nessa perspectiva, a motivação tem sido encarada como uma variável interveniente que, abrangendo elementos internos e situacionais, permite a compreensão do envolvimento individual.

Pesquisas que objetivem descobrir quais são as condições dos contextos sociais que promovem ou prejudicam as potencialidades humanas são valiosas, tanto no aspecto teórico e prático, ampliando o conhecimento formal, quanto para proporcionar ambientes sociais favoráveis ao crescimento e desenvolvimento dos indivíduos. Nas duas últimas décadas, observa-se um aumento acentuado de estudos concentrados na motivação no contexto escolar, objetivando, em sua maioria, encontrar formas de influenciar os alunos a incrementar seu envolvimento em tarefas de aprendizagem (Amabile Hill, Hennessey, Tighe, 1994; Gottfried \& Fleming, 1998; Newby, 1991; Ryan, Connel \& Deci, 1985; Ryan \& Deci, 2000, entre outros).

As investigações têm buscado respostas para problemas tais como, por exemplo, as formas de auxiliar alunos a terminar os trabalhos iniciados, facilitar o desenvolvimento de novas habilidades e de emoções positivas em relação à aprendizagem, melhorar a qualidade dos trabalhos escolares, entre outras. Como resultados positivos desses esforços de estudo, destacase a constituição de estratégias facilitadoras da motivação intrínseca e das modalidades autodeterminadas de motivação extrínseca em situações de aprendizagem escolar, oferecendo uma riqueza de possibilidades para o trabalho cotidiano do professor.

A motivação intrínseca refere-se ao envolvimento em determinada atividade por sua própria causa, por esta ser interessante, envolvente ou, de alguma forma, geradora de satisfação e, sobretudo, com ausência de constrangimentos externos ou internos. Implica ainda em uma orientação pessoal para dominar tarefas desafiadoras, associada ao prazer derivado do próprio processo, evidenciando curiosidade e persistência. Em suma, há motivação intrínseca quando a pessoa envolve-se em determinada atividade sem qualquer recompensa externa ou pressão.

Em relação à aprendizagem escolar, há evidências de que a motivação intrínseca facilita a aprendizagem e o desempenho pois, com essa padrão motivacional, o aluno busca envolver-se em atividades que ofereçam oportunidade para o aprimoramento de suas habilidades, concentra-se nas instruções dadas, procura novas informações, esforça-se para organizar o novo conhecimento de acordo com os seus conhecimentos prévios, além de buscar aplicá-lo a outros contextos. Frente a novas situações de aprendizagem, o aluno intrinsecamente motivado demonstra maior retenção dos conteúdos aprendidos, sente-se confiante, freqüentemente completa as tarefas escolhidas e se mostra satisfeito em realizá-las. O progresso alcançado promove um senso de eficácia em relação à aprendizagem, gerando expectativas positivas de desempenho e realimentando a motivação para aquela tarefa ou atividade.

A motivação extrínseca refere-se à motivação para trabalhar em resposta a algo externo à tarefa, como a obtenção de recompensas materiais ou sociais, de reconhecimento ou objetivando atender aos comandos de outras pessoas. Destaca-se, neste caso, uma avaliação cognitiva do trabalho escolar como um meio dirigido a algum fim extrínseco (Csikszentmihalyi \& Nakamura, 1989; Amabile, Hill, Hennessey \& Tighe, 1994). Estudos recentes têm ampliado a compreensão sobre a motivação extrínseca, sugerindo que esta pode evoluir em um continuum de autodeterminação. Em um extremo do continuum temos a regulação externa, representando o padrão clássico da motivação extrínseca, ou seja, a natureza instrumental do comportamento, voltado para a obtenção ou evitação de eventos externos. No extremo oposto está a regulação integrada, compartilhando os mesmos determinantes e indicadores da motivação intrínseca, ou seja, a flexibilidade cognitiva, o processamento profundo de informações e a criatividade.

Anderson, Blumenfeld, Pintrich, Clark, Marx e Peterson (1995), em seu trabalho sobre a função da Psicologia Educacional na formação de professores, consideram importante identificar e compreender as 
metas e orientações motivacionais dos alunos com o objetivo de ampliá-las e dirigi-las para o aprender, estimulando a aceitação de desafios, a proposição de idéias próprias e criando um clima no qual não se salientem preocupações com notas e avaliações. Além disso, defendem a conscientização dessas orientações pessoais por parte dos futuros professores, possibilitando-lhes uma análise, a partir de fatos da própria experiência, sobre o tipo de motivação que deve ser privilegiado em situações escolares.

Na presente pesquisa foram investigadas as orientações motivacionais de alunos de licenciatura para com a disciplina Psicologia da Educação e os fatores a elas relacionados.

\section{MÉTOD0}

Participaram deste estudo alunos dos cursos de Licenciatura da Universidade Estadual de Londrina ( $\mathrm{N}=246)$, sendo $162(65,85 \%)$ mulheres e $84(34,14 \%)$ homens. Com as informações obtidas sobre a idade formaram-se três faixas etárias, sendo que a Faixa 1 agrupou os alunos até 20 anos (n=92; 37,4\%); a Faixa 2 , de 21 a 30 anos $(n=122 ; 49,6 \%)$; e a Faixa 3, de 31 anos ou mais $(n=32 ; 13 \%)$.

Os critérios utilizados para a seleção da amostra foram: (a) ser aluno de um dos cursos de licenciatura oferecidos pela instituição; (b) já ter cursado ou estar cursando a disciplina Psicologia da Educação. Do total de alunos que responderam ao instrumento, cerca de um terço $(34,9 \%)$ atuava no ensino. Em relação ao sexo, das 162 mulheres, 58 lecionavam (35,80\%); para o grupo masculino, 28 participantes lecionavam (33, $73 \%$ ). Além disso, $37,8 \%$ dos participantes relataram ter forte intenção de trabalhar no ensino após a conclusão do curso, enquanto que $60,66 \%$ destes aceitariam, sem entusiasmo, teriam pouca ou nenhuma intenção de lecionar.

\section{Instrumentos}

A elaboração dos instrumentos para a coleta de dados baseou-se em diversas escalas já referidas na literatura específica (Wentzel, 1989; Amabile \& cols., 1994).

\section{Instrumento 1: Dados demográficos}

Foram levantadas informações sobre sexo, idade, área de trabalho (principalmente para verificar a atuação como professor). Foi incluída também a avaliação da intenção de exercer o magistério após a formatura, com base nos resultados do trabalho de Serow (1994), que indicaram um entusiasmo e compromisso com a idéia de ensinar significativamente maior nos indivíduos que se consideraram vocacionados para o ensino. Além disso, esses professores mostraram-se mais interessados nos problemas inerentes à profissão e foram mais propensos a aceitar as obrigações extras que acompanham o papel de professor. Na presente pesquisa, buscou-se avaliar se as orientações motivacionais e o esforço dos que realmente querem ser professores diferem daqueles que não indicaram ter essa intenção.

\section{Instrumento 2: Avaliação da Motivação Intrín- seca e Extrínseca}

$\mathrm{O}$ instrumento elaborado por Amabile e cols.. (1994), The Work Preference Inventory (WPI) foi traduzido e adaptado. Segundo os próprios autores, esta foi a primeira avaliação voltada para as orientações motivacionais intrínseca e extrínseca, especificamente de populações adultas, incluindo alunos de cursos superiores. Ele é composto de duas escalas de 15 itens cada uma, sendo que as medidas de avaliação da motivação intrínseca apresentam itens referentes à preferência por atividades desafiadoras, autonomia, realização da atividade por sua própria causa e busca de aprofundamento. A escala de avaliação da motivação extrínseca contém itens a respeito da preocupação com capacidade, reconhecimento, ser melhor do que os outros e obtenção de recompensas. Como no instrumento original, os itens foram escritos na primeira pessoa e os participantes deviam assinalar a alternativa que melhor os descrevesse, numa escala de quatro pontos, variando de $1=$ (nunca ou quase nunca verdadeiro para mim) a $4=$ (sempre ou quase sempre verdadeiro para mim).

A análise da homogeneidade dos itens de motivação intrínseca e extrínseca aplicados neste estudo resultou em um a de Cronbach de 0,52 e 0,56, respectivamente.

\section{Instrumento 3: Avaliação do Esforço Alegado}

O questionário buscou avaliar a percepção dos estudantes sobre o grau de esforço despendido na disciplina Psicologia da Educação. Foram elaboradas sete questões baseadas no trabalho de Wentzel (1989). Os itens desta escala de esforço alegado foram formulados na 
primeira pessoa e aos participantes coube assinalar uma alternativa, numa escala de três pontos, variando de "raramente", "algumas vezes" ou "sempre me esforcei".

A análise de homogeneidade dos itens através de análise fatorial resultou num a de Cronbach de 0,77 para a amostra selecionada neste estudo.

\section{Resultados}

A tabela 1 apresenta a estatística descritiva da percepção de desempenho, das médias obtidas pelos par- e ao esforço alegado foram maiores para o sexo feminino.

Outra variável a que se relacionaram os resultados das escalas de motivação intrínseca, extrínseca e esforço alegado foi o fato de os participantes estarem ou não lecionando atualmente. Surgiu diferença significativa em relação ao nível de esforço alegado: $F(1,240)=$ $6,42, p=0,01$ e entre as médias obtidas na escala de motivação intrínseca: $F(1,240)=3,28, p=0,07$. Os estudantes que atuam no ensino obtiveram médias superiores nas duas medidas.

A próxima relação significativa foi entre os esco-

Tabela 1: Estatística descritiva dos escores médios referentes à percepção de desempenho, motivação intrínseca, extrínseca e esforço alegado

\begin{tabular}{lcccc}
\hline & Percepção de Desempenho & Motivação Intrínseca & Motivação Extrínseca & Esforço Alegado \\
\hline $\mathrm{n}$ & 246 & 239 & 234 & 243 \\
Média & 2,58 & 2,01 & 2,01 & 2,02 \\
Mínimo & 1 & 1 & 1 & 1 \\
Máximo & 4 & 3 & 3 & 3 \\
Desvio P & 0,68 & 0,59 & 0,55 & 0,56 \\
\hline
\end{tabular}

ticipantes nas escalas de avaliação da motivação intrínseca, extrínseca e do esforço alegado. A variação do número de observações em cada escala é resultante da eliminação dos escores daqueles que, por algum motivo, deixaram de responder integralmente aos itens nela propostos.

A relação entre os escores médios dos participantes nas medidas de Motivação Intrínseca, Motivação Extrínseca, Esforço Alegado por um lado, e a Idade Cronológica por outro lado, discriminadas as três faixas etárias, apontou, através de análise de variância (ANOVA), para uma diferença significativa para a medida do esforço alegado: $\mathrm{F}(2,240)=7,01, \mathrm{p}=0,01$. Pelo teste de Tukey, Honest Significant Difference (HSD), a comparação das faixas duas a duas indicou que o esforço alegado pela Faixa 3 de idade (31 anos ou mais) foi significativamente maior do que o alegado pelas Faixas 1 (até 21 anos) e 2 (21 a 30 anos). Entre as Faixas 1 e 2 não houve diferença significativa.

$\mathrm{Na}$ comparação entre o sexo dos participantes e as médias obtidas em cada uma das referidas escalas, observou-se diferença estatisticamente significativa com relação à média obtida na escala de motivação extrínseca, com $F(1,232)=4,35, p=0,037$ e em relação ao esforço alegado com $F(1,241)=23,69, p=0,01$, sendo que os escores relativos à motivação extrínseca res médios nas avaliações de motivação intrínseca, extrínseca e esforço alegado e a variável independente, o grau de intenção de atuar no magistério após a conclusão do curso de licenciatura. Houve diferença significativa referente à motivação extrínseca sendo $F(3,226)=3,24, p=0,02$. Neste caso, a análise post hoc de Tukey apontou que, tanto os estudantes que aceitam, como os que querem muito lecionar, apresentaram uma média na escala de avaliação da motivação extrínseca significativamente maior do que os que alegaram não ter tal intenção. Além disso, a mesma análise concluiu por diferença estatisticamente significativa em relação ao esforço alegado, $\operatorname{com} F(3,235)=$ $7,2, p=0,01$, revelando a análise de Tukey que os participantes que assinalaram querer muito lecionar após a conclusão do curso alegaram esforço significativamente maior, comparados aos demais participantes que aceitam lecionar, têm pouca ou nenhuma intenção.

A análise de variância das variáveis demográficas combinadas duas a duas em relação às medidas de Motivação Intrínseca, Extrínseca e de Esforço Alegado apresentaram os resultados significativos a seguir:

(a) conforme apresentado na tabela 2, o total de esforço alegado em relação à combinação das variáveis sexo e idade apontou para diferença estatistica- 
mente significativa, sendo $F(5,237)=7,98, p=0,01$. A análise de Tukey revelou que as mulheres, nas três faixas etárias, relataram um nível de esforço significativamente maior do que o alegado pelo grupo masculino nas Faixas etárias 1 e 2 (mais jovens), sendo que não houve diferença significativa em relação ao grupo masculino na Faixa etária 3 (31 anos ou mais); etária 3 (31 anos ou mais) e que lecionam atualmente foi significativamente maior do que o alegado pelas faixas etárias 1 e 2 (mais jovens) que não atuam no ensino;

(c) os resultados da comparação entre o esforço alegado e a combinação das variáveis "leciona atualmente" e a "intenção de trabalhar no ensino, após

Tabela 2: Médias nas escalas de motivação intrínseca, extrínseca e esforço alegado em relação às variáveis sexo e idade

\begin{tabular}{|c|c|c|c|c|c|c|c|}
\hline \multirow[b]{3}{*}{ Sexo } & \multirow[b]{3}{*}{ Faixa Etária } & \multicolumn{4}{|c|}{ Motivação } & & \\
\hline & & \multicolumn{2}{|c|}{ Intrínseca } & \multicolumn{2}{|c|}{ Extrínseca } & \multicolumn{2}{|c|}{ Esforço } \\
\hline & & Média & $\mathrm{N}$ & Média & $\mathrm{N}$ & Média & $\mathrm{N}$ \\
\hline Masculino & Faixa 1 & 2,79 & 24 & 2,48 & 25 & 2,00 & 25 \\
\hline Masculino & Faixa 2 & 2,85 & 49 & 2,54 & 47 & 2,07 & 49 \\
\hline Masculino & Faixa 3 & 2,91 & 10 & 2,56 & 10 & 2,30 & 10 \\
\hline Feminino & Faixa 1 & 2,77 & 64 & 2,67 & 64 & 2,31 & 67 \\
\hline Feminino & Faixa 2 & 2,81 & 72 & 2,59 & 69 & 2,25 & 71 \\
\hline \multirow[t]{3}{*}{ Feminino } & Faixa 3 & 2,98 & 20 & 2,61 & 19 & 2,52 & 21 \\
\hline & $\mathrm{F}$ & 0,80 & //I/ & 1,31 & //I/ & 7,98 & //// \\
\hline & $\mathrm{P}$ & 0,54 & //// & 0,25 & //I/ & 0,01 & //// \\
\hline
\end{tabular}

(b) houve diferença significativa na comparação entre as médias obtidas na escala de avaliação da motivação intrínseca e a combinação das variáveis idade e o fato de estar lecionando atualmente, $F(4,236)=2,32$, $p=0,04$. Mediante o teste de Tukey, foi observado que os indivíduos mais velhos (Faixa etária 3), que atuam no ensino, apresentaram médias superiores na escala de avaliação da motivação intrínseca, comparadas às médias dos indivíduos mais jovens (Faixa etária 1), que não lecionam. Também foram encontradas diferenças significativas em relação ao esforço alegado, sendo $F(5,236)=3,91, p=0,01$. A análise de Tukey revelou que o nível de esforço alegado pelos indivíduos na Faixa a conclusão do curso", apontaram para diferença significativa, $F(6,230)=4,58, p=0,01$. Revelou a análise de Tukey que os participantes que lecionam atualmente e querem muito continuar no ensino após a formatura apresentaram uma média de esforço maior, comparada com as médias daqueles que não lecionam e alegam nenhuma intenção de atuar no ensino após a formatura. Além disso, a média de esforço alegado pelos indivíduos que não lecionam, mas querem muito atuar no magistério após a conclusão do curso, foi maior comparada às médias daqueles que não lecionam e têm pouca/ nenhuma intenção ou aceitam lecionar sem entusiasmo. Os dados são demonstrados na

Tabela 3: Médias nas escalas de motivação intrínseca, extrínseca e esforço alegado em relação às variáveis leciona e intenção de lecionar

\begin{tabular}{|c|c|c|c|c|c|c|c|}
\hline \multirow[b]{3}{*}{ Lec. } & \multirow[b]{3}{*}{ Intenção de lecionar } & \multicolumn{4}{|c|}{ Motivação } & & \\
\hline & & \multicolumn{2}{|c|}{ Intrínseca } & \multicolumn{2}{|c|}{ Extrínseca } & \multicolumn{2}{|c|}{ Esforço } \\
\hline & & Média & $\mathrm{N}$ & Média & $\mathrm{N}$ & Média & $\mathrm{N}$ \\
\hline $\operatorname{Sim}$ & não pretendo & 2,87 & 3 & 2,56 & 4 & 2,50 & 4 \\
\hline $\operatorname{Sim}$ & pouca intenção & 2,97 & 11 & 2,62 & 12 & 2,24 & 12 \\
\hline $\operatorname{Sim}$ & aceito sem muito entusiasmo & 2,82 & 26 & 2,64 & 25 & 2,25 & 27 \\
\hline Sim & quero muito & 2,91 & 43 & 2,64 & 40 & 2,36 & 42 \\
\hline Não & não pretendo & 2,91 & 12 & 2,23 & 10 & 1,99 & 12 \\
\hline Não & pouca intenção & 2,75 & 36 & 2,55 & 36 & 2,14 & 36 \\
\hline Não & aceito sem muito entusiasmo & 2,69 & 56 & 2,65 & 55 & 2,10 & 55 \\
\hline \multirow[t]{3}{*}{ Não } & quero muito & 2,87 & 47 & 2,59 & 47 & 2,38 & 50 \\
\hline & $\mathrm{F}$ & 1,42 & $/ / / /$ & 1,83 & $/ / / /$ & 4,58 & $/ / / /$ \\
\hline & $\mathrm{p}$ & 0,19 & $/ / / /$ & 0,08 & $/ / / /$ & 0,01 & /III \\
\hline
\end{tabular}


(d) a comparação das médias obtidas na avaliação do esforço alegado com combinação das variáveis sexo e o fato de estar lecionando atualmente apontou para diferença estatisticamente significativa, com $F(3,238)=10,16, p=0,01$. Revelou a análise de Tukey que, independentemente do fato de estar lecionando, o nível de esforço alegado pelo grupo feminino foi significativamente maior que o alegado pelo grupo masculino, não havendo, no entanto, diferença significativa entre o esforço alegado pelo grupo masculino que leciona e o alegado pelo grupo feminino que não atua no ensino;

(e) as médias na avaliação do esforço, comparadas à combinação das variáveis sexo e intenção de lecionar após a conclusão do curso, apontaram para diferença significativa, sendo $F(5,231)=7,18, p=0,01$. De acordo com análise de Tukey, o nível de esforço alegado pelo grupo feminino que indica querer muito lecionar foi significativamente maior do que o nível de esforço alegado pelo grupo masculino que não tem intenção, tem pouca ou aceita lecionar sem entusiasmo. Apenas em relação ao grupo masculino que afirma querer muito lecionar, o teste revelou que a média de esforço alegado foi significativamente maior, comparada à do grupo masculino que não revelou tal intenção. No grupo feminino, observou-se uma média de esforço alegado significativamente maior para os que querem muito lecionar, comparados aos que não têm intenção;

(f) houve diferença significativa nas médias obtidas na escala de avaliação da motivação extrínseca em relação a combinação de variáveis sexo e intenção de lecionar, $\operatorname{com} F(5,231)=2,28, p=0,02$. Apontou a análise de Tukey que a média de motivação extrínseca das mulheres que aceitam ou querem muito lecionar foi significativamente maior do que a obtida pelo grupo masculino que assinalou nenhuma intenção de atuar no ensino.

(h) a comparação entre o esforço alegado com a combinação das variáveis idade e intenção de lecionar demonstrou diferença estatisticamente significativa $F(6,227)=3,77, p=0,01$. A análise de Tukey indicou que o nível de esforço alegado pelos sujeitos da Faixa etária 3 (31 anos ou mais) que querem muito lecionar foi significativamente maior do que o alegado pelas Faixas de idade 1 e 2 (mais jovens) que afirmaram ter nenhuma, pouca intenção ou aceitarem sem entusiasmo o fato de lecionar após conclusão do curso. A análise também apontou para diferença significativa em relação à motivação intrínseca, $F(6,223)=2,47, p=0,01$, não sendo reveladas, através da análise de Tukey, diferenças dentro ou entre os pares de comparações.

\section{Discussĩo}

As comparações entre as diversas variáveis que compuseram o presente estudo revelaram aspectos importantes acerca da motivação dos alunos de licenciatura, além de fornecerem indicativos para o encaminhamento da disciplina Psicologia Educacional e para organização dos referidos cursos. De modo geral, podemos considerar que algumas características dos alunos estão relacionadas à maior motivação para a disciplina. As mulheres, os alunos mais velhos, aqueles que atuam no ensino ou que têm clara intenção de ser professores revelaram-se mais motivados.

A informação sobre a pouca intenção dos alunos em atuar no ensino não é novidade. Nos últimos 30 anos, ainda que os discursos oficiais coloquem a educação como meta primordial, assistimos ao rebaixamento salarial progressivo do magistério de forma generalizada, transformando os cursos de formação em opções cada vez menos atraentes, sendo procurados por alunos que, em sua maioria, estudam no período noturno e trabalham em funções não relacionadas à área de educação. Além disso, a escolha em alguns casos é feita porque as licenciaturas representam a possibilidade de garantir um diploma de nível superior, exigido pelo mercado de trabalho cada vez mais seletivo e, em outros casos, a intenção dos alunos não é trabalhar no ensino, mas focalizam o bacharelado para tornar-se, por exemplo, biólogos, químicos, matemáticos, historiadores etc. Demo, 1992; Goulart, (1997); Saviani (1984) comentam ainda que a desvalorização dos cursos de licenciatura é evidente, inclusive dentro das próprias instituições de ensino superior no Brasil, sendo consideradas muitas vezes como as "filhas pobres" das universidades.

Soluções para este problema não parecem simples, mas trazê-lo à discussão nas diversas disciplinas dos cursos de licenciatura pode propiciar a conscientização dos alunos para a necessidade de assumir efetivamente a profissão e, como resultado, buscar uma formação mais sólida.

A medida do esforço alegado pelos participantes em relação à disciplina Psicologia da Educação foi a variável preferencialmente relacionada aos fatores demográficos. Vale ressaltar que tal medida 
corresponde a um indicativo motivacional, ou seja, denota o envolvimento do sujeito para com a disciplina. No entanto, a palavra esforço pode ter significados variados para os indivíduos, de acordo com diversos fatores ligados ao desafio da tarefa. Assim, para alguns estudantes, esforçar-se muito em determinada disciplina pode significar comparecer às aulas, responder às solicitações do professor, estudar nas vésperas das avaliações, enquanto que, para outros, o esforço pode indicar leituras cuidadosas, busca de aprofundamento nos diversos temas abordados, organização das novas informações com conhecimentos anteriores, etc. Segundo Blumenfeld (1992), tal diversidade na avaliação dos alunos é decorrente da valorização daquilo que está sendo obtido ou aprendido na situação. Frente a isto, é esperado que os estudantes relutem em encarar os trabalhos desafiadores quando é criada uma norma implícita no grupo sobre o que se configura um nível de esforço adequado. Neste quadro, muitas vezes, o professor é levado a ceder às reações negativas geradas pela situação e diminuir o nível de exigência das atividades apresentadas.

Como base nessas proposições, podemos inferir que a desvalorização das licenciaturas nas instituições de ensino superior pode estar presente nas percepções dos alunos em relação às disciplinas pedagógicas. Assim, empregar determinado grau de esforço nessas disciplinas pode representar para os alunos um empenho acima das expectativas culturalmente vivas em nosso meio. Uma vez que está implícito o nível de esforço ideal, tentativas de superá-lo acarretariam resistência por parte dos estudantes, levando também os docentes a organizarem suas atividades de acordo com esse padrão estabelecido.

O problema acima descrito destaca a necessidade de que o planejamento da disciplina Psicologia Educacional seja apresentado e discutido com os futuros professores de forma clara, prevendo as atividades a serem desenvolvidas, os objetivos a serem alcançados e os procedimentos avaliativos a serem utilizados. Assim, transparecerá aos estudantes que assumir a carreira do magistério exige uma ampla formação, respaldada nos fundamentos da educação, os quais incluem os conhecimentos da Psicologia Educacional, e que só pode ser obtida com envolvimento e esforço.

A questão do gênero pode auxiliar a compreensão da maior motivação apresentada pelo grupo feminino. Historicamente, no Brasil e em outras sociedades, a carreira de ensino sempre foi caracterizada como área de trabalho feminino. Sendo a opção por uma determinada função articulada com a apreciação das atividades a serem desenvolvidas e em conformidade com a identidade social e com o papel sexual do indivíduo (Lighbody \& Durndell, 1996), pode-se supor que, para o grupo feminino, esta escolha dá-se de maneira natural, posto que as coincidências entre aqueles papéis e as exigências da futura profissão são convergentes. No caso da opção masculina pelo magistério, as características do esteriótipo do papel de professor não são necessariamente vinculadas às características profissionais delegadas socialmente aos homens. Em decorrência, a revelação por parte do grupo masculino de um grande envolvimento em uma disciplina componente do quadro de formação pedagógica do curso, representaria a anuência destes indivíduos para com as características profissionais ou área de interesse que não são socialmente dirigidas ou valorizadas para os homens. Lighbody e Durndell (1996) assinalam que esses padrões de valorização social são de tal forma incorporados aos valores individuais que passam a não ser percebidos conscientemente como valores externos, mas que influenciam as percepções, as decisões ou outras atribuições individuais.

A mesma linha de análise pode auxiliar a compreensão das médias superiores apresentadas pelo grupo feminino na avaliação da motivação extrínseca. O sistema educacional, como apontado por diversas pesquisas (Larson \& Rafaelli, 1989; Csikszentmihalyi, 1992; entre outros), acentuam predominantemente o aspecto extrínseco da aprendizagem, sendo que o ingresso na escola sinaliza para as crianças que o importante é obter boas notas, responder satisfatoriamente às exigências do professor e obter as recompensas por esses comportamentos, realizar os melhores trabalhos para ser reconhecido etc. Fortalecendo essa tendência da escola, a sociedade, mais especificamente a família, desde cedo transmite às crianças o que é esperado delas e esses valores são gradativamente interiorizados pelos indivíduos à medida em que vão se desenvolvendo (Stipek, 1998). Desta forma, os alunos chegam ao curso superior socializados para buscar essas metas, ou seja, orientados extrinsecamente. Como para o grupo feminino a profissão do magistério é uma opção socialmente esperada, tanto seu envolvimento quanto a busca de recompensas ou de reconhecimentos externos diferem positivamente em comparação com o grupo masculino, ou seja, refletem uma maior concordância das mulheres para com as demandas educacionais. 
Mesmo estando as questões do gênero ligadas às escolhas profissionais, expectativas e relações sociais, o tema tem sido pouco considerado nos cursos de formação de professores (Grossi, 1994). Paraíso (1997) argumenta que essa ausência no currículo formal não impede que o problema esteja presente no cotidiano das salas de aula, manifestando-se através da ação de alunos e professores, criando e fortalecendo esteriótipos do que é aceitável para cada papel social.

A cada geração, o mundo social e histórico mostra-se diferente, mesmo que permaneçam certos atributos e, nesse contexto, homens e mulheres transformam-se social e biologicamente (Paraíso, 1997). Reconhecendo essa mobilidade, podemos vislumbrar alternativas de solução para os problemas relacionados ao gênero a partir da formação de professores. Incluir nos currículos os conhecimentos já construídos na área (Bernardes, 1997), e possibilitar reflexões e análises sobre crenças e atitudes pode contribuir para a formação de um profissional atento à questão e capaz de criar um clima em sala de aula que desvaneça a rigidez dos papéis socialmente aceitos ou convencionados.

A idade dos alunos participantes foi outra variável importante em termos motivacionais. Ingressar em um curso superior com idade acima da média esperada pode significar o desfecho de uma trajetória envolvendo diversas tentativas de opções profissionais ou pessoais (outros cursos de graduação, trabalho em outras áreas, casamento, família, etc.), possibilitando a estes alunos uma maior definição acerca das suas expectativas e possibilidades de desempenho profissional ou, como poderíamos denominar, uma escolha madura.

Observou-se também que o fato de o participante estar atuando no ensino implicou em um maior envolvimento na disciplina Psicologia Educacional. Provavelmente, o contato direto com os problemas reais do cotidiano da sala de aula propicia a percepção das disciplinas pedagógicas pelos alunos como sendo um território no qual a compreensão e a superação desses problemas podem ser viabilizadas. Neste sentido, reforçam-se as premissas do trabalho de Anderson e cols. (1995), a respeito da Psicologia Educacional. Indicam esses autores que uma contribuição efetiva da

\section{REFERÊNCIAS}

Amabile, T. M., Hill, K. G., Hennessey, B. A., \& Tighe, E. M. (1994). The Work Preference Inventory assessing referida disciplina na formação de professores deve ser buscada através de uma articulação com a realidade educacional, isto é, o contato dos alunos com problemas educacionais reais permitirá que os conteúdos abordados sejam considerados elementos facilitadores da compreensão e da elaboração de estratégias de intervenção. Desse modo, a proposta daqueles autores de se promover situações de contato com os problemas reais, com a possibilidade de uso efetivo de diferentes instrumentais teóricos da psicologia, nos cursos aqui contemplados torna-se ainda mais pertinente, dada a baixa porcentagem de estudantes que trabalham com a educação.

\section{Conclusĩo}

A opção pelo magistério é um elemento essencial para a motivação dos estudantes de licenciatura. A afirmação pareceria óbvia demais não fossem as características peculiares destes cursos. A desvalorização, a questão do gênero, a preferência pelo bacharelado ou a simples obtenção de um diploma de curso superior tornam os estudantes diferenciados em termos do envolvimento nos conteúdos das disciplinas de formação.

A Psicologia Educacional construiu, ao longo dos anos, um corpo de conhecimentos que possibilitam a compreensão e a melhoria do ensino. Cabe a nós, professores da disciplina, constante atualização e valorização desse conhecimento acumulado. Isto pode ser efetivado através da aproximação com as expectativas e necessidades dos alunos, do planejamento de tarefas autênticas, que representem a complexidade do ensino e que possam desenvolver nos futuros professores uma perspectiva psicológica útil, isto é, que possibilite a observação e a busca de solução para os problemas de sala de aula, embasadas nas diversas abordagens teóricas que compõem a área (Bzuneck, 1999). Desse modo, podemos contribuir para ampliar suas metas e orientações motivacionais e, finalmente, auxiliar na formação de profissionais preparados para enfrentar os inúmeros desafios que a educação nos apresenta.

intrinsic and extrinsic motivational orientations. Journal of Personality and Social Psychology, 6(5), 950-967.

Anderson, L. M., Blumenfeld, P., Pintrich, P., Clark, C., Marx, 
R. \& Peterson, P. (1995). Educational Psychology for teachers: reforming our curses, rethinking our roles. Educational Psychologist, 30(3), 143-157.

Bernardes, N. (1997). Gênero e educação: construção, debate e polêmica. Educação e Realidade, 22(1), 225-231.

Blumenfeld, P. C. (1992). Classroom learning and motivation: clarifying and expanding goal theory. Journal of Educational Psychology, 84(3), 272-281.

Brophy, J. (1983). Conceptualizing student motivation. Educational Psychologist, 18 (3), 200-215.

Bzuneck, J. A. (1999). A Psicologia Educacional e a formação de professores: tendências contemporâneas. Psicologia Escolar e Educacional, 3(1), 41-52.

Csikszentmihalyi, M. \& Nakamura, J. (1989). The dinamics of intrinsic motivation: a study of adolescents. Em C. Ames \& R. Ames (Eds.), Research on Motivation in Education. Goals and Cognitions, 3, 249-277. New York: Academic Press.

Csikszentmihalyi, M.(1992). A psicologia da felicidade. São Paulo: Saraiva.

Demo, P. (1994). Crise dos paradigmas na educação superior. Educação Brasileira, 16(32), 97-100.

Gottfried, A. E., Fleming, J. S. (1998). Role of cognitively stimulating home environment in children's academic intrinsic motivation. Child Development, 69 (5), 14481461.

Goulart, A T. (1996). Os cursos de licenciatura: desafios, propostas e alternativas. Educação Brasileira, 18(36), 97-109.

Grossi, M. P. (1994). O masculino e o feminino na educação. Em E. P. Grossi \& Bordin (Eds.) Paixão de Aprender ,252-258, Petrópolis, Vozes.

Larson, R., Ham, M. \& Rafaelli, M. (1989). The Naturance of Motivated Attention. In M. Maehr \& R. Ames (Eds.), Advances in motivation and Achievement: Motivation Enhancing Enviromments 6,45-80. Connecticut: Jai Press Inc.
Lightbody, P. \& Durndell, A (1996). The masculine image of careers in science and tecnology: fact or fantasy? British Journal of Educational Psychology, 231-246.

Newby, T. J. (1991) Classroom motivation: strategies of firstyear teachers. Journal of Educational Psychology, 83 (2), 195-200.

Paraíso, M. A (1997). Gênero na formação docente: campo de silêncio no currículo. Cadernos de Pesquisa, 102, 241-255.

Parra, N. (1984). Cursos de Licenciatura: Uma Proposta de Reforma. Revista da Faculdade de Educação de São Paulo, 10 (2), 191-198.

Ryan, R. M., Connell, J. P. \& Deci, E. L. (1985). A motivational analysis of self-determination and self-regulation in education. In C. Ames \& R. Ames (Eds.), Research on Motivation in Education, 2, 16-31. New York: Academic Press.

Ryan, R. M., Deci, E. (2000). Self-determination theory and the facilitation of intrinsic motivation, social development, and well-being. American Psychologist, 55(1), 68-78.

Saviani, D. (1984). Ensino público e algumas considerações sobre universidade. São Paulo: Cortez.

Serow, R. (1994). Called to teach: a study of highly motivated preservice teachers. The Journal of Research and Development in Education, 27(2), 65-72.

Stipek, D. J. (1998). Motivation to learn. from theory to pratice. Englewood Cliffs: Prentice-Hall.

Wentzel, K. R. (1989). Adolescent classroom goals, standards for performance and academic achievement: an interactionist perspective. Journal of Educational Psychology, 81(2), 131-142.

Recebido em: 06/06/00

Revisado em:12/03/01

Aprovado em:27/12/01 